Article

\title{
Facile Synthesis of Solvent Sensitive Fluorinated Graphene Nanoscrolls and Application as a Cathode Material for Lithium Ion Batteries
}

\author{
Guozhong Liu ${ }^{1}$, Weihong Wan ${ }^{2, *}$, Jinsheng Cheng ${ }^{2,}$, Litianlun $\mathrm{Xu}^{1}$, Zhihe $\mathrm{Chen}^{3}$, \\ Ali Chen ${ }^{3}$, Xueqi Zeng ${ }^{2}$ and Jinying $\mathrm{Ke}^{2}$ \\ 1 School of Electrical Engineering \& Intelligentization, Donguan University of Technology, Dongguan, \\ 523808, China \\ 2 School of Ying-Dong Food Sciences and Engineering, Shaoguan University, Shaoguan, 512005, China \\ 3 Central Laboratory, Guangdong Pharmaceutical University, Guangzhou 510006, China \\ * Correspondence: chengjins@gmail.com; weihonggd@gmail.com; Tel.: +86-751-812-0167
}

\begin{abstract}
In this work, fluorinated graphene nanoscrolls (FGN) were synthesized via facile chemical methods under simple and mild conditions. Interestingly, the formation of the featured FGN was significantly solvent sensitive. Experimental results indicated that in the presence of aprotic solvent, for example, $\mathrm{N}, \mathrm{N}$ dimethylformamide (DMF), the reaction system inclined to form the interesting FGN nanostructures. The structure and morphology of the prepared FGN were detailed characterized by atomic force microscopy (AFM), transmission electron microscopy (TEM), scanning electron microscopy (SEM) and X-ray diffraction (XRD) etc. The obtained FGN was used as a cathode material for primary lithium ion batteries with superior discharge specific capacity (eg. 979.3 $\mathrm{mAhg}^{-1}$ ), stable discharge platform and high energy density (eg. $2287.9 \mathrm{Wh} \mathrm{kg}^{-1}$ ), which fosters it a high density, low cost and durable candidate for cathode material for lithium ion batteries..
\end{abstract}

Keywords: Linear fluorinated graphene nanoscrolls, solvent sensitive, synthesis, cathode material

\section{Introduction}

With the digital development of military and aviation technology (for example, satellites, spacecraft, space stations, deep space exploration landers, orbit transfer vehicles and drones etc.) [1-2], the requirements for various high-performance power sources are getting higher and higher ${ }^{[3-4]}$. For example, the weight of each component of the power sources was required to be as light as possible to increase the effective load, the power supply was also demanded to be able to discharge at a large current and provide higher power to meet the rapid response of military or aviation equipment ${ }^{[5]}$.

Lithium/carbon fluoride $\left(\mathrm{Li}_{1} / \mathrm{C}_{1} \mathrm{~F}_{\mathrm{x}}\right)$ primary battery is a kind of solid cathode lithium battery first commercialized [6], and its theoretical mass specific energy can achieve to $2180 \mathrm{Wh} / \mathrm{kg}[7]$, which was much higher than widely used $\mathrm{Li} / \mathrm{SOCl}_{2}$ primary battery $\left(1470 \mathrm{Whkg}^{-1}\right)^{[8]}$ and $\mathrm{Li} / \mathrm{MnO}_{2}$ candidate $\left(1005 \mathrm{Whkg}^{-1}\right)^{[9]}$. The featured lithium/carbon fluoride primary battery has the advantages of a stable discharge platform, excellent safety and storage performance, together with wide operating temperature range ${ }^{[10-11]}$. However, due to the poor intrinsic conductivity of the cathode material fluorocarbon and the slow electrode dynamics process, the lithium/carbon fluoride primary battery has a larger polarization performance during the discharge processes. Meanwhile, the layered structure of the described lithium/carbon fluoride is not conducive to the lithium ion electrochemical 
reaction [12]. Therefore, the lithium/carbon fluoride battery is limited to discharge at a low rate, leading to voltage hysteresis at the beginning of the discharge. At the same time, there is a large amount of heat consumption during the discharge process of $\mathrm{Li} / \mathrm{C}_{1} \mathrm{~F}_{\mathrm{x}}$ primary battery, which affects the performance of the battery obviously and severely restricts the application of lithium/carbon fluorine batteries [13].

Facing such difficulties, necessary modification for the lithium/carbon fluoride cathode material to enhance the electrochemical performance was expected. In the recent year, different attempts, for example, $\mathrm{MnO}_{2}$ doped $\mathrm{Li} / \mathrm{C}_{1} \mathrm{~F}_{x}{ }^{[9]}$, multiple-walled carbon nanotubes modified $\mathrm{Li} / \mathrm{C}_{1} \mathrm{~F}_{\mathrm{x}}{ }^{[14]}$, polyaniline coated $\mathrm{Li} / \mathrm{C}_{1} \mathrm{~F}_{\mathrm{x}}{ }^{[15]}$, conductive polymer coated $\mathrm{Li} / \mathrm{C}_{1} \mathrm{~F}_{\mathrm{x}}{ }^{[16]}$ and modification to fluorinated graphene ${ }^{[17]}$ etc., were commonly used as electrode material to improve the rate performance of fluorinated carbon materials in lithium batteries. Among which, significant attention had paid to the electrode material of fluorinated graphene ${ }^{[18]}$, which was derived from $\mathrm{C}_{1} \mathrm{~F}_{\mathrm{x}}$. The electrochemical performance of the derived fluorinated graphene was significantly better than the $\mathrm{C}_{1} \mathrm{~F}_{\mathrm{x}}$ candidate. This was mainly due to the fact that the edge and base surface of the cathode electrode material of fluorinated graphene contains a large number of C-F covalent bonds after modification to fluorinated graphene. Therefore, when the modified fluorinated graphene was used as cathode materials of lithium batteries, the lithium ion diffusion rate was significantly improved [19]. Importantly, the superiority of stable discharge platform, excellent safety and storage performance and wide operating temperature range of the $\mathrm{Li} / \mathrm{C}_{1} \mathrm{~F}_{\mathrm{x}}$ electrode material was reserved.

Until now, few report had paid to the preparation of fluorinated graphene nanoscrolls (FGN) or nanorods and corresponding application as a cathode material for lithium ion batteries. In this work, a novel FGN nanostructure with different fluorine doping contents were prepared successfully from fluorinated graphite $\left(\mathrm{C}_{1} \mathrm{~F}_{\mathrm{x}}\right)$ substituted with different fluorine contents by modified Hummers method. Interestingly, the FGN prepared in this work exhibited interesting solvent regulation characteristics. In aprotic solvents, the featured FGN can obtain readily in the reaction system. While in protonic solvents, the reaction system inclined to formation of nanosheets structured fluorinated graphene instead of the featured FGN. Meanwhile, further investigation on FGN as cathode material for primary lithium batteries was also carried out. Investigations revealed that the solvent-regulated FGN prepared in this work exhibited excellent electrochemical characteristics and large power density when used as a cathode electrode material for primary lithium batteries.

\section{Results and discussion}

\section{Preparation and Characterization of Solvent Sensitive FGN}

To date, few reports had been demonstrated on the FGN preparation and application, which might introduced C-F semi-ionic bonds and a large number of defects on the basis of pristine graphene to accelerate the conduction of lithium ions and promote the transmission of ions and electrons as a new high-efficiency lithium battery cathode material. In this work, we used graphite fluoride $\left(\mathrm{C}_{1} \mathrm{~F}_{\mathrm{x}}\right)$ as the starting material. By using modified Hummers method [20-21], fluorinated graphene oxide (FGO) nanosheets with abundant oxygen-containing groups was obtained. The obtained FGO intermediate was reduced by hydrazine hydrate under reflux conditions, giving commonly reported fluorinated graphene nanosheets (Scheme 1) ${ }^{[22]}$.

Interestingly, the formation of the prepared fluorinated graphene nanostructure showed significantly solvent sensitive characteristics. Experimental results indicated that in the presence of protic solvent, for example, ethanol, methanol or water etc., wrinkled fluorinated graphene nanosheets with a thickness of about $0.9 \mathrm{~nm}$ were given readily (determined by atomic force microscopy (AFM) analysis, Fig. 1a). While when the obtained wrinkled fluorinated graphene nanosheets were re-dispersed in aprotic solvent, for example, N,N dimethylformamide (DMF), dimethyl sulphoxide (DMSO) or N-methylpyrrolidone (NMP) etc., by further ultrasound for 15 min and aging for 24-72h, the reaction system inclined to form the interesting FGN nanostructures (Fig. $1 \mathrm{~b}-\mathrm{c})$ instead of nanosheets structured fluorinated graphene showed in Fig. 1a. 
TEM image illustrated in Fig. 1b showed a view of the prepared nanocomposites with clear evidence of nanoscroll structure (FGN). Meanwhile, the prepared FGN had approximate width range of 150-300 nm and length range of 1.0-3.0 $\mu \mathrm{m}$. The SEM image (Fig. 1c) further corroborated the nanoscroll structures of the prepared FGN. The width range and length range observed in Fig. 1c was in accordance with the results observed in Fig. $1 b$.
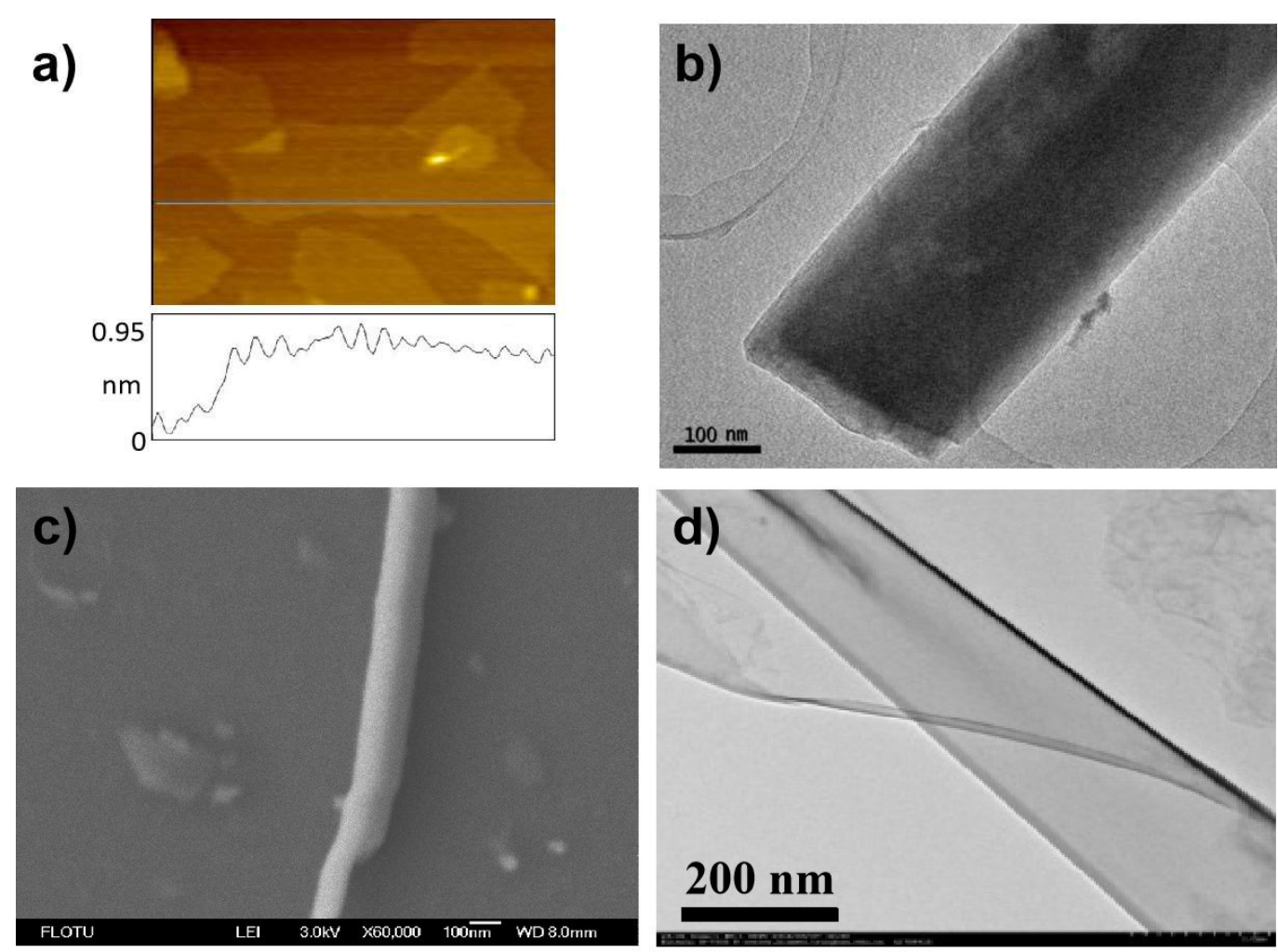

Figure 1. AFM image depth profile of fluorinated graphene dispersed in ethanol, supported on mica substrate, size $2.0 \times 1.8 \mu \mathrm{m}$ a), TEM image of fluorinated graphene nanoscrolls (FGN) in DMF b), SEM images of FGN dispersed in DMF c), both TEM and SEM images showed the rolled structure in the synthetic process of FGN (typically fulorine ratio of $\mathrm{C}_{1} \mathrm{~F}_{\mathrm{x}}, \mathrm{x}=0.8$ ) in $\mathrm{DMF}$ 


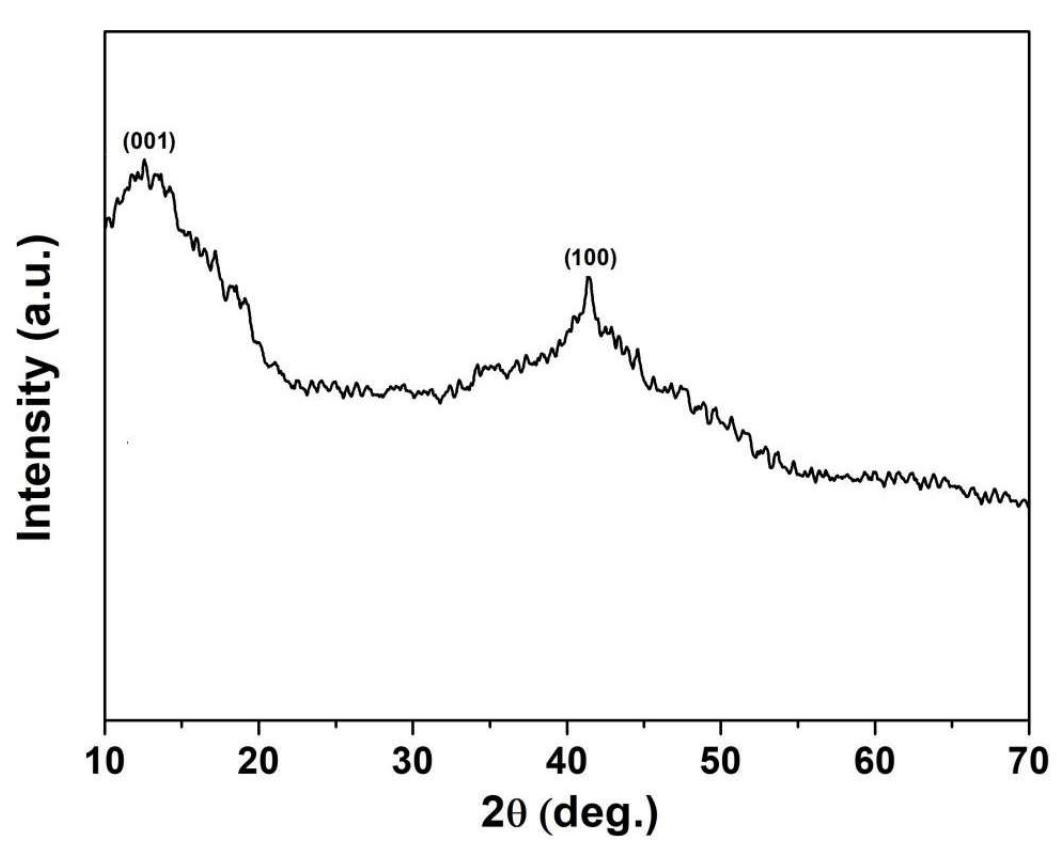

Figure 2. AFM image and depth profile of aLGN on mica substrate, size $2.1 \times 2.1 \mu \mathrm{m}$.

The obtained FGN nanocomposites were also characterized by XRD etc. From the XRD patterns showed in Fig. 2, we can observe that the prepared FGN nanocomposites gave a characteristic peak at $2 \theta=12.9^{\circ}$, which was in conformity with literature results of fluorinated graphene peak (001) [20], confirming that the prepared FGN nanocomposites possessed the structure characteristics of fluorinated graphene. Peak at $2 \theta=42.0^{\circ}$ can be assigned the graphene peak (100) (corresponding to $\mathrm{C}-\mathrm{C}$ bonds in graphene structure [22]), indicating that the prepared FGN nanocomposites were partially restored to ordered crystal structure of graphene.

\section{Possible mechanism for the formation of Solvent Sensitive FGN}

The possible mechanism for the featured FGN nanocomposites formation could be described by the procedures showed in Scheme 1. Firstly, FGO nanosheets were prepared by modified Hummers method (step 1). In this work, that graphite fluoride with different fluorine substituted ratios was used as the starting material. As shown in Scheme 1, after reduction treatment illustrated in step 2 of Scheme 1, fluorinated graphene nanosheets with 2D nanosheet morphology was obtained readily.

Due to containing of abundant $\mathrm{C}-\mathrm{F}$ and $\mathrm{C}-\mathrm{H}$ bonds, the prepared fluorinated graphene nanosheets described in Scheme 1 evoked abundant hydrogen bonds. Meanwhile, the prepared fluorinated graphene nanosheets held strong $\pi-\pi$ interaction with the special fused aromatic structure or benzene rings in the the same or different nanosheet. It's noteworthy that when such hydrogen bonds force were occurred in aprotic solvent, for example, DMF, dimethyl sulfoxide (DMSO) and N-methyl pyrrolidone (NMP) etc. Due to deficiency of protons in the solvent, the hydrogen bonds in fluorinated graphene inclined to form in the same fluorinated graphene nanosheet or multiple fluorinated graphene nanosheets (between $\mathrm{F}$ atoms and $\mathrm{H}$ atoms, further multiple effects led to formation of the nanoscroll structure finally) instead of hydrogen bonds between fluorinated graphene nanocomposites and solvent moleculars. While when protonic solvent, for example, $\mathrm{H}_{2} \mathrm{O}$, ethanol, methanol etc., each fluorinated graphene nanosheet was surrounded by many protonic solvent moleculars (e. g. $\mathrm{H}_{2} \mathrm{O}$ ), besides hydrogen bonds in the same fluorinated graphene nanosheet or different ones, abundant hydrogen bonds between solvent moleculars and fluorinated graphene nanosheets would form, which can drive the prepared nanocomposites to form the commonly nanosheet structured fluorinated graphene. 


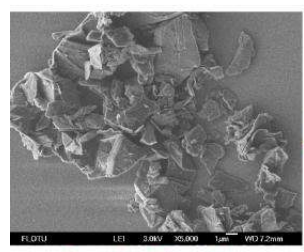

Graphite Fluoride

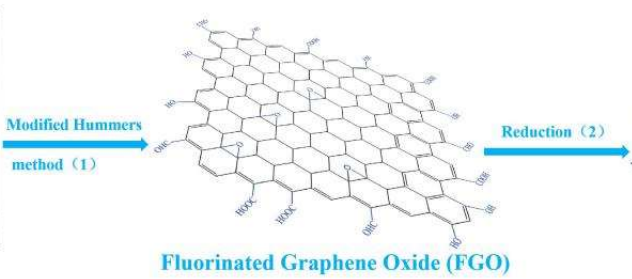

Fluorinated Graphene Oxide (FGO)

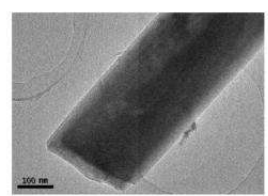

Fluorinated Graphene Nanoscrolls(FGN)

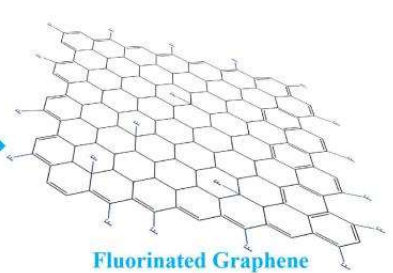

Fluorinated Graphene \begin{tabular}{l|l}
$\begin{array}{l}\text { electrostatic interaction } \\
\text { hydrogen bonding }\end{array}$ & Self-curling effect
\end{tabular} (3)

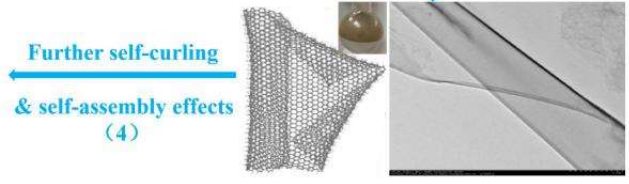

Self-curling Intermediate (SCI)

Scheme 1. Mechanism for the synthesis of the FGN.

By synergistic effects with plentiful hydrogen bonds and electrostatic interactions, the self-curling intermediate (SCI) was obtained readily. As shown in SEM image in Scheme 1 (step 3), the multiple forces can drive the SCI intermediate rolled gradually. It's exciting that such transient process was captured by SEM analysis successfully (Scheme 1).

Moreover, synergistic effects of plentiful hydrogen bonds, $\pi-\pi$ interactionand and electrostatic interactions in singled or multiple nanosheets during the the SCI formation processes (step 3) would further accumulate and accelerate, leading to heavily rolled structure of intermediate SCI. After aging treatment for 24-72h, the featured FGN nanocomposites could be obtained readily (step 4 of Scheme 1). In such procedures, associated self-assembly procedures would also occur, which was helpful for the formation of FGN nanocomposites (Scheme 1).

Further investigations confirmed the solvent sensitive characteristics of the prepared fluorinated graphene. As shown in TEM image of Fig. $3 \mathrm{a}$, when the prepared nanocomposites was dispersed in ethanol, a typical protonic solvent, due to multiple forces described above, the reaction system formed nanosheet structured fluorinated graphene. On a comparison study, when aprotic solvent, for example, DMF, DMSO etc, was used, nanoscrolls structured fluorinated graphene instead of nanosheet structured one, can be given, which can be observed by TEM images (Fig. 3b-c). Interestingly, when nanoscrolls structured FGN showed in Fig.3b was re-dispersed in ethanol, as examined by TEM image (Fig. 3d), the reaction system inclined to shift back to nanosheet structured fluorinated graphene, clearly exhibiting the interesting solvent sensitive fluorinated graphene nanocomposites. 
a)

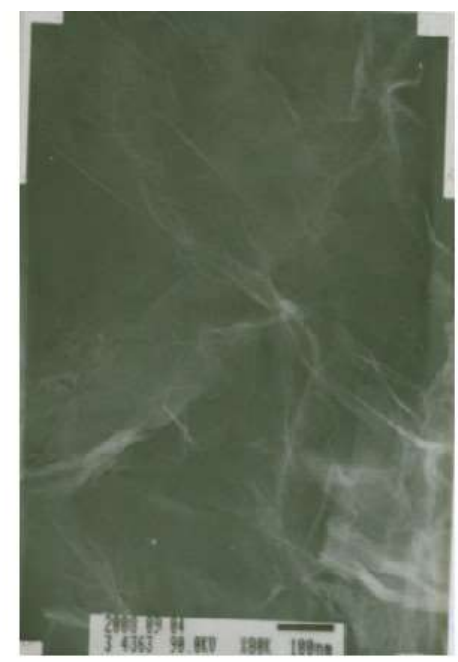

c)

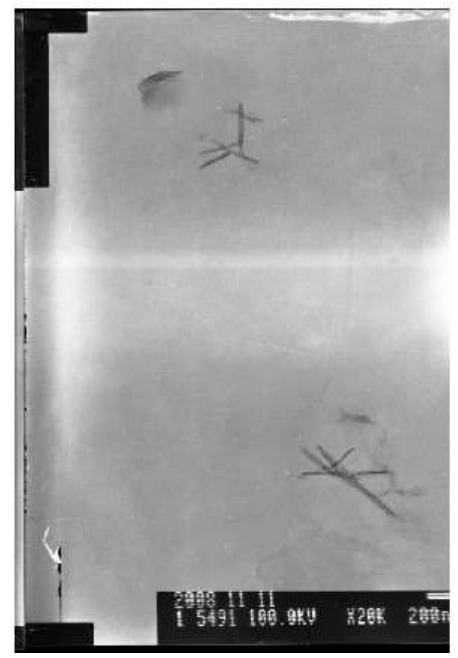

b)

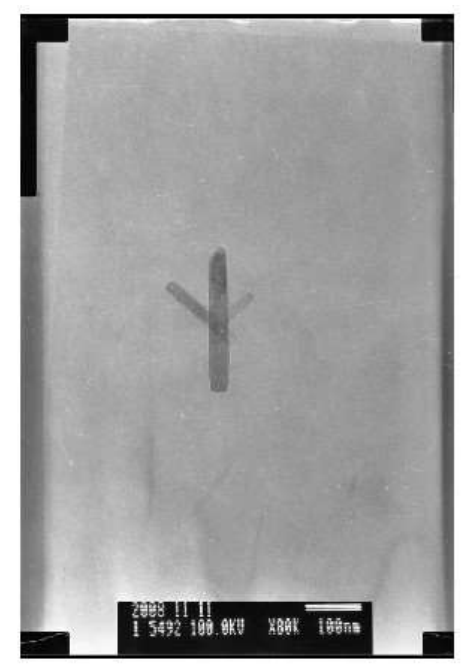

d)

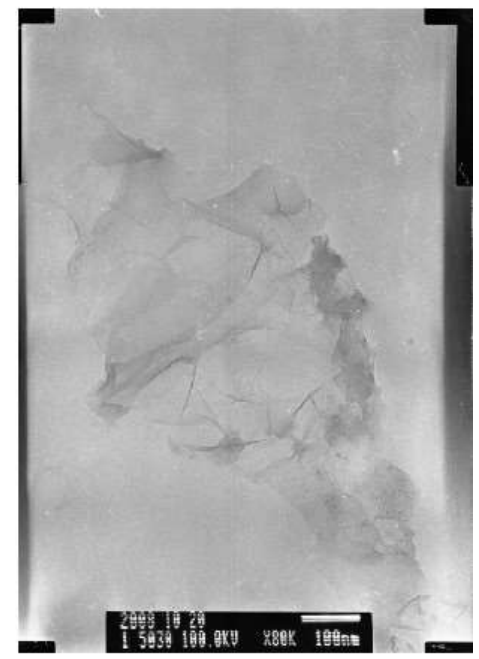

Figure 3. Fabrication of fluorinated graphene nanocomposites with different morphologies: solvent tuned structures of fluorinated graphene: TEM image of fluorinated graphene dispersed in ethanol a), TEM image of fluorinated graphene in DMF b), TEM image of fluorinated graphene dispersed in DMSO c), FGN showed in image b) was filtered and re-dispersed in ethanol, showing nanosheet structure.

\section{Electrochemical performance of the prepared FGN as a cathode material for lithium ion batteries}

The electrochemical performance of the prepared FGN as a cathode material for lithium ion batteries was tested in this work. Fig. 4 showed the constant current discharge curve of the prepared FGN with different fluorine substitution ratios as the cathode electrode material, which was then assembled into a button battery. As shown in Figure 4a-b, when FGN-1.1 or FGN-0.8 was used, the increase in discharge current density led to a decrease in average discharge voltage and specific capacity. This should be caused by the Ohmic polarization effect. At the same time, we can also observe intuitively that the electrochemical properties of the prepared FGN were closely related to the fluorine content. Under the same current density, the discharge specific capacity was directly proportional to the fluorine content of the prepared FGN. For example, FGN-1.1, the specific discharge capacity of FGN-1.1 was measured at three different current densities with below values: $979.3 \mathrm{mAhg}^{-1}(50 \mathrm{~mA}), 802.6 \mathrm{mAhg}^{-1}(100 \mathrm{~mA}), 670.7 \mathrm{mAhg}^{-1}(200 \mathrm{~mA})$ (Fig. 4a), which was much higher than the corresponding discharge specific capacity values when FGN-0.8 was determined: 902.8 $\mathrm{mAhg}^{-1}(50 \mathrm{~mA}), 765.1 \mathrm{mAhg}^{-1}(100 \mathrm{~mA})$ and 648.2.9 $\mathrm{mAhg}^{-1}(200 \mathrm{~mA})$ (Fig. 4b). This might on account of that FGN with higher fluorine content possessed lower conductivity, resulting in a larger Ohmic polarization effects and a decrease of discharge voltage. What's more, experimental results indicated 
that the prepared FGN nanocomposites, eg. FGN-1.1, had stable discharge platform range from 50$600 \mathrm{~mA}$. And the discharge voltages of FGN-1.1 were relatively lower than those of lower fluorine substituted FGN (for example, FGN-0.8). As shown in Fig. 4a (FGN-1.1), the discharge voltages were $2.42(50 \mathrm{~mA})$ and $2.19(200 \mathrm{~mA})$, respectively, which were also obviously higher than the contradistinctive data showed in Fig. 4b (FGN-0.8, $2.68(50 \mathrm{~mA}), 2.36(200 \mathrm{~mA}))$. Detailed discharge performances of different fluorine substituted FGN revealed that the highest discharge specific capacity $\left(979.3 \mathrm{mAhg}^{-1}\right)$ can be achieved when the fluorine substitution ratio of FGN was 1.1 at the current density of $50 \mathrm{~mA}$.
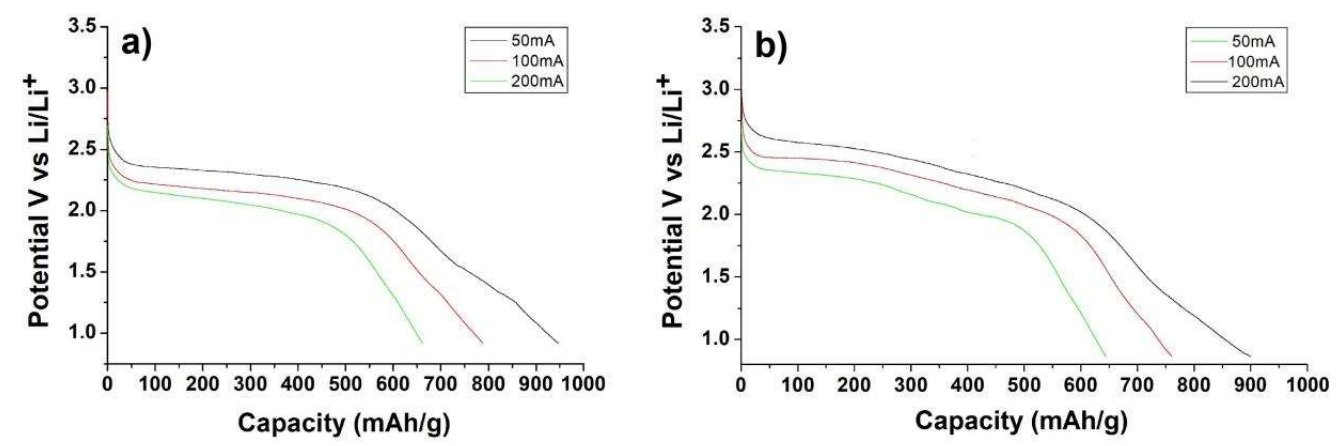

Figure 4. Charge and discharge properties of FGN with different fulorine ratio (C1Fx): $x=1.1 \mathrm{a}), \quad \mathrm{x}=0.8 \mathrm{~b})$.

Figure 5. Ragone plots of the prepared FGN with different fluorine substituted contents

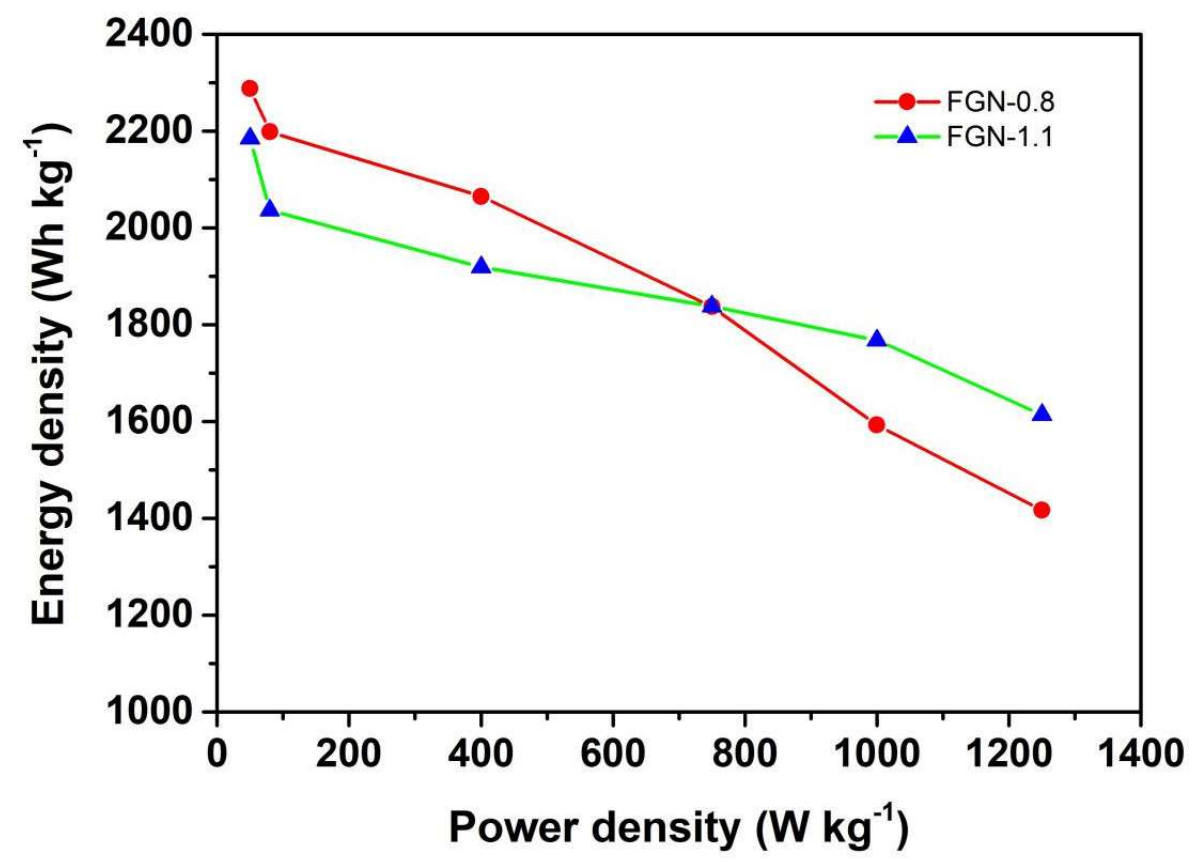

On the other hand, a further comparative energy density analysis of of the prepared FGN were carried out in this work. As shown in Fig. 5, due to decrease of discharge voltage and specific capacity, the energy density of the FGN decreased as the power density increased. It's noteworthy that at low energy density, the prepared FGN $(x=0.8)$ showed good electrochemical performance, and the energy density reached the maximum value $\left(2287.9 \mathrm{Wh} \mathrm{kg}^{-1}\right)$ at a current of $50 \mathrm{mAg}^{-1}$. It is gratifying that the measured value was significantly higher than the theoretical energy density of the $\mathrm{Li}_{1} / \mathrm{C}_{1} \mathrm{~F}_{x}$ galvanic cell mentioned above (2180 Wh kg-1), indicating that modification of carbon fluoride $\left(\mathrm{C}_{1} \mathrm{~F}_{\mathrm{x}}\right)$ to fluorinated graphene led to significantly improved of energy density. Meanwhile, at high value of 
energy density, the prepared FGN with higher fluorine contents ( $x=1.1$ instead of 0.8$)$ exhibited higher energy density. As shown in Fig. 5, when the current was $1250 \mathrm{mAg}^{-1}$, the energy density can even reach $1613.8 \mathrm{Wh} \mathrm{kg}^{-1}$, which was obvious higher than the value (1416.5 $\mathrm{Wh} \mathrm{kg}^{-1}$ ) of FGN with lower fluorine contents $(\mathrm{x}=0.8)$ under the same current density.

\section{Methods}

\section{Materials and characterization}

Graphite fluoride with different fluoride contents (99.99\%, 325 mesh) were purchased from Alfa Aesar. Hydrazine hydrate (analytically pure) was purchased from Sigma Aldrich. All solvents and other reagents were purchased from Beijing Chemicals Co. Ltd. as analytical-grade products.

The powder X-ray diffraction (XRD) measurements of the samples were recorded on a Bruker D8Advance $X$-ray powder diffractometer using $\mathrm{Cu} \mathrm{K} \alpha$ radiation $(\lambda=1.5406 \AA)$ with scattering angles $(2 \theta)$ of 8-60'. JEOL JEM 1200EX and JEOL JEM 2010 transition electronic microscopy were used for transmission electron microscopy (TEM) analysis and high-resolution transmission electron microscopy (HRTEM) analysis at an accelerating voltage of $100 \mathrm{kV}$, respectively. Samples were prepared by placing one drop of an ethanol suspension of FGN or other nanomaterials onto a copper grid (3 mm, 200 mesh) coated with carbon film. A JSM-7401 scanning electron microscopy (SEM) operated at $20 \mathrm{kV}$ was used to analyze the sample.

\section{Typical procedures for the preparation of FGN}

Fluorinated graphene oxide (FGO, showed in Scheme 1) was prepared by modified Hummers method [21-22]. In a typical procedures, graphite fluoride powder $\left(1.5 \mathrm{~g}, 325\right.$ mesh, $\mathrm{C}_{1} \mathrm{~F}_{\mathrm{x}}, \mathrm{x}=0.5,0.6,0.8$ and 1.1 etc.) was put into a mixture of $12 \mathrm{ml}$ concentrated $\mathrm{H}_{2} \mathrm{SO}_{4}, 2.5 \mathrm{~g} \mathrm{~K} 2 \mathrm{~S}_{2} \mathrm{O}_{8}$ and $2.5 \mathrm{~g} \mathrm{P}_{2} \mathrm{O}_{5}$. The solution was heated to $80{ }^{\circ} \mathrm{C}$ and kept stirring for $5 \mathrm{~h}$ by using oil-bath. In a next step, the mixture was cooled to room temperature and diluted with deionized water $(500 \mathrm{ml})$ overnight. Then, the product was obtained by filtering using 0.2 micron nylon film and dried naturally. The pre-oxidized graphite was then re-oxidized by Hummers method. Pretreated graphite powder was put into $0{ }^{\circ} \mathrm{C}$ concentrated $\mathrm{H}_{2} \mathrm{SO}_{4}(120 \mathrm{ml})$. Then, $15 \mathrm{~g} \mathrm{KMnO}_{4}$ was added gradually under stirring and the temperature of the mixture was kept to be below $20^{\circ} \mathrm{C}$ by ice-bath. Successively, the mixture was stirred at $35{ }^{\circ} \mathrm{C}$ for $4 \mathrm{~h}$, and then diluted with $250 \mathrm{~mL}$ deionized water by keeping the temperature under $50{ }^{\circ} \mathrm{C} .700 \mathrm{ml}$ deionized water was then injected into the mixture followed by adding $20 \mathrm{ml} 30 \%$ $\mathrm{H}_{2} \mathrm{O}_{2}$ drop by drop. The mixture was filtered and washed with $1: 10 \mathrm{HCl}$ aqueous solution (1 L) to remove metal ions followed by $1 \mathrm{~L}$ of deionized water to remove the acid. The resulting solid was dried in air and diluted to make FGO dispersion $(0.5 \% \mathrm{w} / \mathrm{w})$. Finally, it was purified by dialysis for one week to remove the remaining metal species, finally, the sample of FGO was obtained by filtration and dried in vacuum for $24 \mathrm{~h}$ at $60{ }^{\circ} \mathrm{C}$. Successively.

Firstly, the sample of FGO was re-dispersed in water $(0.5 \mathrm{mg} / \mathrm{ml})$ and ultrasoniced for $15 \mathrm{~min}$, then hydrazine hydrate $(1 \mathrm{ml})$ was added gradually to the mixture. The suspension was stirred at $80{ }^{\circ} \mathrm{C}$ for $24 \mathrm{~h}$. Finally, the dispersion was cooled down and filtered, the solid sample was collected after thoroughly washing with deionized water and ehanol until the impurities can be removed completely. The sample was vacuum-dried at $38.6^{\circ} \mathrm{C}$ for $24 \mathrm{~h}$, affording the reduced composite: nanosheet structured fluorinated graphene (detected by AFM, showed in Fig. 1a) can be afforded.

On a comparison study, the obtained solid of FGO was re-suspended in DMF to form a dispersion of $1 \mathrm{mg} / \mathrm{ml}$. The mixture $(100 \mathrm{ml})$ was ultrasoniced for $15 \mathrm{~min}$, then hydrazine hydrate $(1 \mathrm{ml})$ was added gradually to the mixture. The suspension was stirred at $80{ }^{\circ} \mathrm{C}$ for $24 \mathrm{~h}$. Finally, the dispersion was cooled down and filtered, the solid sample was collected after thoroughly washing with deionized water and DMF until the impurities can be removed completely. The sample was vacuumdried at $38.6^{\circ} \mathrm{C}$ for $24 \mathrm{~h}$, affording the reduced composite: FGN(detected by TEM analysis, Fig. 1b) . 
9 of 11

\section{Electrochemical analysis}

In this work, after preparation of button half-cells, cyclic voltammetry $(\mathrm{CV})$ test was used to study the surface chemical reactions of the prepared electrodes during charge and discharge procedures, so that to explore the mechanism of electrodes reaction. The CV scan voltage range was 0.01-3.0V, and the scan rate is $0.5 \mathrm{mVs}^{-1}$. Meanwhile, the capacity, coulombic efficiency, cycle and rate characteristics of FGN were studied through constant current charge and discharge tests.

\section{Conclusion}

In this paper, a novel fluorinated graphene nanoscrolls (FGN) with different fluorine doping contents were synthesized via facile chemical methods under simple and mild conditions. Interestingly, the formation of the featured FGN was significantly solvent sensitive. Experimental results indicated that in the presence of aprotic solvent, for example, N,N dimethylformamide (DMF), the reaction system incline to form the interesting FGN nanostructures. The structure and morphology of the prepared FGN were detailed characterized by AFM, TEM, SEM and XRD etc. Further investigation on FGN as cathode material for primary lithium batteries was also carried out. Investigations revealed that the solvent-regulated FGN prepared in this work had excellent electrochemical characteristics and large power density when used as a positive electrode material for primary lithium batteries. Detailed discharge performances of different fluorine substituted FGN indicated that the highest discharge specific capacity $\left(979.3 \mathrm{mAhg}^{-1}\right)$ and stable discharge platform range from 50-600 mA were observed when the fluorine substitution ratio of FGN was 1.1 at a current density of 50mA. On the other hand, FGN with lower fluorine content (the fluorine substituted ratio was 0.8 instead of higher ones) can reach the maximum energy density (2287.9 Wh $\left.\mathrm{kg}^{-1}\right)$ at a current of $50 \mathrm{mAg}^{-1}$, which fosters it a high density, low cost and durable candidate for cathode material for lithium ion batteries.

Data availability. The data that support the plots within this paper are available from the corresponding author upon reasonable request.

Acknowledgments. This work was financially supported by the Guangdong National Science Foundation of China (No. 2019A1515011163), Research start-up funds of DGUT (GC300502- 10), Guangdong University Innovation Team Project (2019KCXTD017), Innovation Projects of General Colleges and Universities of Guangdong Province (2018KTSCX- 203) and 2020 Guangdong General University \& Shaoguan New Crown Pneumonia Epidemic Prevention and Control Research Special Project (2020KZDZX1190).

Author contributions. Liu G. Z., Xu L. L. and Cheng J. S. collected and reviewed data, and discussed results. Cheng J. S. and Wan W. H. designed the study and wrote the paper, Chen Z. H.and Chen A. L. performed statistical analyses. All authors approved the submission.

Conflicts of Interest. The authors declare no conflict of interest. The founding sponsors had no role in the design of the study; in the collection, analyses, or interpretation of data; in the writing of the manuscript, and in the decision to publish the results. 


\section{References}

[1] Spitzer, Cary, Uma Ferrell, and Thomas Ferrell, eds. Digital avionics handbook. CRC press, 2017.

[2] Moore, Mark. "Aviation Frontiers-On Demand Aircraft." 10th AIAA Aviation Technology, Integration, and Operations (ATIO) Conference. 2010.

[3] Leuchter J, Bloudicek R, Blasch E, et al. Overview of Electrical Backup Power Supply Systems for Air Navigation Facilities based on the Energy Storage Technology[C]//2019 IEEE/AIAA 38th Digital Avionics Systems Conference (DASC). IEEE, 2019: 1-9.

[4] Boukoberine, Mohamed Nadir, Zhibin Zhou, and Mohamed Benbouzid. "A critical review on unmanned aerial vehicles power supply and energy management: Solutions, strategies, and prospects." Applied Energy 255 (2019): 113823.

[5] Kuo K. Military Innovation and Technological Determinism: British and US Ways of Carrier Warfare, 19191945[J]. Journal of Global Security Studies, 2020.

[6] Jones J P, Jones S C, Billings K J, et al. Radiation effects on lithium CFX batteries for future spacecraft and landers[J]. Journal of Power Sources, 2020, 471: 228464.

[7] Zhou R, Li Y, Feng Y, et al. The electrochemical performances of fluorinated hard carbon as the cathode of lithium primary batteries[J]. Composites Communications, 2020, 21: 100396.

[8] Chavillon B, Blanc L, Mayousse E, et al. Specific liquid cathode battery: U.S. Patent Application 15/823,198[P]. 2018-5-31.

[9] Yang K, Shan Z, Liu X, et al. Study on modifying the Li/MnO2 battery by mixing with the carbon fluoride[J]. Energy Storage, 2020, 2(4): e128.

[10] Liang H, Lawrence N, Jones T, et al. Methods of producing a lithium carbon fluoride primary battery: U.S. Patent Application 16/408,503[P]. 2019-8-29.

[11] Xuerong Q, Ji G U O, Juan M I. Test Research of High Specific Energy Lithium Carbon-Fluoride (Li/CFx) Batteries as Power Sources for Deep Space Exploration[J]. Journal of Deep Space Exploration, 2020, 7(1): 87-92.

[12] von Aspern N, Röschenthaler G V, Winter M, et al. Fluorine and Lithium: Ideal Partners for HighPerformance Rechargeable Battery Electrolytes[J]. Angewandte Chemie International Edition, 2019, 58(45): 15978-16000.

[13] Liu Y, Jiang L, Wang H, et al. A brief review for fluorinated carbon: synthesis, properties and applications[J]. Nanotechnology Reviews, 2019, 8(1): 573-586.

[14] Li Y, Wu X, Liu C, et al. Fluorinated multi-walled carbon nanotubes as cathode materials of lithium and sodium primary batteries: effect of graphitization of carbon nanotubes[J]. Journal of Materials Chemistry A, 2019, 7(12): 7128-7137.

[15] Li L, Zhu L, Pan Y, et al. Integrated polyaniline-coated CF $x$ cathode materials with enhanced electrochemical capabilities for Li/CF x primary battery[J]. Int. J. Electrochem. Sci, 2016, 11: 6838-6847.

[16] Nakajima, Tsuyoshi, and Henri Groult, eds. Fluorinated materials for energy conversion. Elsevier, 2005.

[17] Zhong G, Chen H, Huang X, et al. High-power-density, high-energy-density fluorinated graphene for primary lithium batteries[J]. Frontiers in chemistry, 2018, 6: 50.

[18] Peng S, Yan S, Wang N, et al. Fluorinated graphene/sulfur hybrid cathode for high energy and high power density lithium primary batteries[J]. RSC advances, 2018, 8(23): 12701-12707.

[19] Bobnar J, Lozinšek M, Kapun G, et al. Fluorinated reduced graphene oxide as a protective layer on the metallic lithium for application in the high energy batteries[J]. Scientific reports, 2018, 8(1): 1-10.

[20] Chang H, Cheng J, Liu X, et al. Facile synthesis of wide-bandgap fluorinated graphene semiconductors[J]. 
Chemistry-A European Journal, 2011, 17(32): 8896-8903.

[21] Cheng J, Zhong S, Wan W, et al. Novel Graphene/ $\operatorname{In}_{2} \mathrm{O}_{3}$ Nanocubes Preparation and Selective Electrochemical Detection for L-Lysine of Camellia nitidissima Chi[J]. Materials, 2020, 13(8): 1999.

[22] Yu B , Huang Q, Liu Y, et al. Fabrication of composite biofibres based on chitosan and fluorinated graphene for adsorption of heavy metal ions in water[J]. The Journal of the Textile Institute, 2019, 110(3):426-434.

[23] Liou Y J, Huang W J. Quantitative analysis of graphene sheet content in wood char powders during catalytic pyrolysis[J]. Journal of Materials Science \& Technology, 2013, 29(5): 406-410. 